\title{
Influence of enzymatic treatments on white wine composition
}

\author{
E.C. Scutaraşu ${ }^{1}$, V.V. Cotea ${ }^{1}$, C.E. Luchian ${ }^{1}$, L.C. Colibaba ${ }^{2}$, N. Katalin² ${ }^{2}$, R. Oprean ${ }^{3}$, and M. Niculaua \\ ${ }^{1}$ University of Agricultural Sciences and Veterinary Medicine "Ion Ionescu de la Brad", Iaşi, Romania \\ 2 "Iuliu Haşieganu" University of Medicine and Pharmacy, V. Babeş Street, Cluj Napoca, Romania \\ ${ }^{3}$ Research Center for Oenology, Romanian Academy - Iaşi Branch, Romania
}

\begin{abstract}
Most biochemical reactions involved in the wine-making process are catalyzed by enzymes. The use of enzymes of exogenous origin in wine production is due to the numerous technological and economical advantages demonstrated over time in the winemaking process. Understanding the important role played by enzymes in wine making technology contributes to the development of optimization strategies for the production process to improve the final quality of the wine. In order to accomplish this study, the influence of five oenological preparations with pectolytic and $\beta$-glucosidases enzymes types on the volatile compounds of white wines obtained from Fetească regală variety was analyzed by monitoring their evolution during the alcoholic fermentation to the final product. Wine samples have been physically and chemically analysed $\left(\mathrm{pH}\right.$, acidity, alcoholic strength, density, malic acid, lactic acid, sugar content, $\mathrm{SO}_{2}$, total dry extract and non-reducing extract) according to OIV Standards. Separation and identification of flavor compounds was performed using an Agilent 7890 gas chromatograph coupled to a $5975 \mathrm{C}$ inert XL EI/CI MSD spectrophotometer. The organoleptic evaluation of wines was made according to a wide range of sensory descriptors. An important evolution of volatile compounds during fermentation was observed, depending on the type of enzyme administered, compared to the control sample. Enzymatic treatments did not significantly affect the physico-chemical composition of the wines obtained. The chromatic parameters of the wine samples varied according to the type of enzyme applied. The results of the study showed a significant influence of the enzymes on the organoleptic characteristics of the wines. Therefore, the aromatic quality of a wine is directly proportional to the chemical composition of the grapes and to the technology.
\end{abstract}

\section{Introduction}

Enzymes are biological catalysers used in winemaking technology for their numerous advantages on the product quality. These compounds may come from indigenous microflora of grapes, yeasts or other bacteria correlated with wine cellar, vinification conditions or vineyard [1].

The wine-maker can enrich the activity of these endogenous enzymes by using commercial products, usually obtained by fermenting pure culture of selected microorganism [2]. Pectinases, proteases and glycosidases are some of the enzymes produced by yeast, that present significance in oenology for their technological actions and their participation to aroma constitution. Pectinolytic enzymes have the ability to cleave long pectin chains into shorter and more soluble chain segments, that facilitate pressing operation of the grapes and clarification of musts, enhace the extraction of color and aroma compounds and facilitate the filtration of resulted wine. These enzymes can also facilitate the stabilisation of the wines, due to the enzymatic hydrolysis of the protein into smaller molecules, containing more nitrogen. The aroma compounds concentration of wines can be increased by glycosidases, which hydrolyse nonvolatile glycosidic precursors of the grapes. Yeasts are also important producers of enzymes [3]. Saccharomyces cerevisiae, considered the fundamental wine yeast, is not appreciated as a considerable producer of extracellular proteases, lipases or proteolytic enzymes [4]. The final quality of wines is influenced by a multitude of interactions between chemical compounds. During alcoholic fermentation, grapes derivates are converted by yeast into a range of sensorial relevant metabolites that gives wine its vinous character [5]. Commercial enzymes are utilized in wine-making technology for diverse biotransformation reactions from pre-fermentation stage pending alcoholic fermentation, post-fermentation and wine aging period [6]. Understanding the important role of enzymes in winemaking technology contributes to the development of strategies to optimize the production process, improving the wine's composition and its sensory characteristics [7].

The purpose of this experiment is to investigate the influence of enzymes pre-treatments on the physicochemical parameters, chromatic characteristics and aromatic profile.

\section{Materials and methods}

\subsection{Grapes samples and winemaking}

The grapes representing Fetească Regală variety were harvested in autumn of 2018, from Iasi vineyard, with approximately $250 \mathrm{~g} / \mathrm{L}$ sugar content. After destemming and crushing operations, the must was transferred into $50 \mathrm{~L}$ glass vessels. A dose of $20 \mathrm{~g} / \mathrm{hL}$ Saccharomyces yeast (Levulia esperide, AEB) and $30 \mathrm{~g} / \mathrm{hL}$ yeast nutrient (FERMO PLUS CH, AEB) (previously dissolved in warm

(C) The Authors, published by EDP Sciences. This is an Open Access article distributed under the terms of the Creative Commons Attribution License 4.0 (http://creativecommons.org/licenses/by/4.0/). 
must) was inoculated in each variant. For this experiment, five commercial enzymes based on pectolytic and $\beta$-glucosidases activity were added to musts before alcoholic fermentation, thus contributing to the release of aroma compounds (Endozym Thiol $\AA$, AEB - V1; Endozym $\beta$-Split ${ }^{\circledR}$, AEB - V2; Zymovarietal aroma G®, SODINAL - V3; Endozym Ice ${ }^{\circledR}$, AEB - V4; Zimarome ${ }^{\circledR}$, BSG WINE -V5 and no enzyme- V6), at doses of $3 \mathrm{~g} / \mathrm{hL}$ (all enzyme preparations were diluted with must 1:10) and $3 \mathrm{~mL} / \mathrm{hL}$ respectively. 6 variants were obtained. The fermentation was carried out at $16-18^{\circ} \mathrm{C}$ for about one week. For this experiment, samples were collected at two days differences during the alcoholic fermentation and stored in the freezer until the analysis. When the alcoholic fermentation was finished, each variant was filtered using sterile membrane filter followed by sulphitation (to preserve wine from microbiological damage) and bottled. The wine samples were stored under controlled condition and analysed after three months.

\subsection{Chemicals}

Standard reagents and pure commercial substances were purchased from Merck KGaA (Germany).

\subsection{Methods of analyses}

Chromatic parameters were analysed using a Specord UV-VIS spectrophotometer, in accordance to the Commission Internationale d'Eclairage (CIE, 1976), using aspects of specific qualities of visual sensation: clarity, tonality, chromatic parameters, saturation, luminosity, hue [8]. CIELab space system is based on a sequential or continuous Cartesian representation of 3 orthogonal axes: $\mathrm{L}^{*}, \mathrm{a}^{*}$, and $\mathrm{b}^{*}$. The vertical axis noted with $\mathrm{L}^{*}$ (clarity) measures from 0 - completely opaque, to $100-$ completely transparent, and parameters " $+\mathrm{a}^{*}$ " red, " $-\mathrm{a}^{*}$ " green, " $+\mathrm{b}$ *" yellow, "-b" blue were registered [9].

Standard physico-chemical parameters were reviewed according to International Organization of Vine and Wine Compendium methods of analysis [8]. Each variant was analysed for: total and volatile acidity, alcoholic strength, $\mathrm{pH}$, density, total sugar, free and total sulphur dioxide, malic and lactic acid, total dry extract and non-reducing extract.

Sensory characteristics are important for the quality of wines. The wine samples were assessed for sensory characteristics by 10 tasters according to a wide range of sensory descriptors. The parameters were evaluated with ratings from 0 to 5 , and the mean was registered in the tables. The sensory descriptors of samples were comparatively expressed in a chart.

Separation and identification of flavour compounds was performed using an Agilent 7890 gas chromatograph coupled to a $5975 \mathrm{C}$ inert XL EI/CI MSD spectrophotometer. The column used for the analysis was HP-5MS fused silica capillary column $(30 \mathrm{~m} \cdot 0.25 \mathrm{~mm}$ I.D., $0.25 \mu \mathrm{m}$ with a split ratio of 50:1 and inlet temperature was set at $250^{\circ} \mathrm{C}$. The MSD was used at $70 \mathrm{eV}$ in the electron impact mode (EI), using the mass range from 50 to $800 \mathrm{Da}$ at $150^{\circ} \mathrm{C}$. Peak identification of the aroma components was achieved by comparison of mass spectra with the mass spectral data collection from Wiley275 and NIST05a libraries.

Sample extraction: In a $15 \mathrm{~mL}$ glass tube, $5 \mathrm{~mL}$ of wine sample and $1 \mathrm{~mL}$ of dichloromethane were added, and the mixture was mechanically agitated for 45 minutes. The extract was separated from the aqueous phase by centrifugation at $7800 \mathrm{rpm}$ for 10 minutes. This process was repeated twice, the extraction was performed after 20 minutes. The two organic phases were mixed and injected in the GC-MS.

\section{Results and discussions}

The present article highlights a comparative investigation on the action of enzymes on the quality of wines.

\subsection{Effects of enzymatic pre-treatment on basic parameters of resulted wine}

Table 1 shows the physical-chemical parameters of wines. The alcoholic strength of a wine is dependent of the total sugar content of the origin grapes. The obtained samples were dry wines with over $12.7 \%$ vol. Alcoholic strength of wines was not influenced by the type of administrated enzymes.

Acidity and $\mathrm{pH}$ are relevant to the sensory perception of wine, essentially defining its structure and equilibrium. Excessive acidity increases the perception of sourness since low acidity decreases the flavour balance. Acid content is relevant for preservation and is responsible for sensory features of wines. Its content in wine may be influenced by grape variety, ripeness, climatic conditions, wine-making process, wine storage and $\mathrm{pH}$ [9]. No significant differences were recorded in total and volatile acidity of wines, which means that enzymes have no influence on this indicator. The total dry extract shows values between 19 and $19.6 \mathrm{~g} / \mathrm{L}$. The content of non-reducing extract of most Romanian wines varies between 13 and $35 \mathrm{~g} / \mathrm{L}$, according to grape variety, health conditions, applied technology and wine treatments [10]. The analysed samples registered values of non-reducing dry extract between 17.6 and $17.9 \mathrm{~g} / \mathrm{L}$.

\subsection{Chromatic characteristics}

Colour is a primary attribute by which the consumer assesses wine and food. Chromatic parameters of analysed wines is presented in Table 2.

Analysed samples registered a high clarity, with more yellow and red shades, except V1 that presented more green and yellow shades of colour. Parameter " $a *$ " (green/red component) had the highest value on V6 (control sample) and the lowest on V1 sample (Endozym Thiol®). The highest values of " $b$ " " (blue/yellow component) were registered in the case of V1 sample (Endozym Thiol ${ }^{\circledR}$ ), and the lowest in V3 (Zymovarietal aroma $\left.G^{\circledR}\right)$. Some differences were recorded for " $L^{*}$ " that corresponded to brightness characteristics. It was expressed by a less green/red and more yellow colour of wine. The red pigment indicates the presence of low but still visible amounts of anthocyanins. Enzymatic treatment influenced chromatic parameters of analysed samples to varying degrees. The majority of wines treated with enzymatic preparations were characterized by the decrease in $\mathrm{a}^{*}$ compared to the control sample, a less intense green colour and more red colour respectively. Also, all samples treated with enzymes were evaluated by an increase in $b^{*}$, that means more yellow colour. 
Table 1. Physical-chemical parameters of wines.

\begin{tabular}{|l|l|l|l|l|l|l|l|l|l|l|l|l|}
\hline Sample & $\begin{array}{l}\text { Total } \\
\text { Acid } \\
\mathrm{g} / \mathrm{L} \\
\mathrm{C}_{4} \mathrm{H}_{6} \mathrm{O}_{6}\end{array}$ & $\mathrm{pH}$ & $\begin{array}{l}\text { Alcoholic Malic } \\
\text { strength } \\
\text { \% vol. }\end{array}$ & $\begin{array}{l}\text { V.A. } \\
\text { Acid } / \mathrm{L} \\
\mathrm{C}_{2} \mathrm{H}_{4} \mathrm{O}_{2}\end{array}$ & Density & $\begin{array}{l}\text { Total } \\
\text { Sugar } \\
\mathrm{g} / \mathrm{L}\end{array}$ & $\begin{array}{l}\text { Lactic } \\
\text { Acid }\end{array}$ & $\begin{array}{l}\text { Free } \\
\mathrm{SO}_{2} \\
\mathrm{mg} / \mathrm{L}\end{array}$ & $\begin{array}{l}\text { Total } \\
\mathrm{SO}_{2} \\
\mathrm{mg} / \mathrm{L}\end{array}$ & $\begin{array}{l}\text { T.D.E. } \\
\mathrm{g} / \mathrm{L}\end{array}$ & $\begin{array}{l}\text { N.E. } \\
\mathrm{g} / \mathrm{L}\end{array}$ \\
\hline V1 & 4.2 & 3.28 & 12.8 & 2.7 & 0.13 & 0.9908 & 1.8 & 0 & 23 & 56 & 19.6 & 17.8 \\
\hline V2 & 4.2 & 3.36 & 12.7 & 2.9 & 0 & 0.9909 & 1.7 & 0.1 & 23 & 56 & 19.6 & 17.9 \\
\hline V3 & 4.2 & 3.3 & 12.8 & 2.9 & 0.08 & 0.9907 & 1.6 & 0.2 & 25 & 58 & 19.3 & 17.7 \\
\hline V4 & 4.2 & 3.28 & 12.7 & 2.8 & 0.14 & 0.9907 & 1.4 & 0.2 & 25 & 58 & 19 & 17.6 \\
\hline V5 & 4.3 & 3.27 & 12.7 & 2.8 & 0.1 & 0.9907 & 1.3 & 0.2 & 23 & 58 & 19 & 17.7 \\
\hline V6 & 4.2 & 3.28 & 12.8 & 2.9 & 0.12 & 0.9907 & 1.4 & 0.3 & 25 & 56 & 19.3 & 17.9 \\
\hline
\end{tabular}

Table 2. Chromatic parameters of wines.

\begin{tabular}{|l|c|c|c|c|c|c|c|c|c|}
\hline \multirow{2}{*}{ Sample } & \multirow{2}{*}{ L Clarity } & \multicolumn{2}{|c|}{ Cromaticity } & \multirow{2}{*}{ Crome C } & \multirow{2}{*}{ Tonality $\mathbf{H}$} & \multirow{2}{*}{ Lightness } & \multirow{2}{*}{ Hue } & $\Delta \mathbf{E}$ & $\Delta \mathbf{H}$ \\
\cline { 3 - 10 } & & $\mathbf{a}^{*}$ & $\mathbf{b}^{*}$ & & & & & & \\
\hline V1 & 98.3 & -0.12 & 5.61 & 5.61 & -88.82 & 0.12 & 3.32 & 2.72 & 1.92 \\
\hline V2 & 96.9 & -1.44 & 4.92 & 5.13 & -73.70 & 0.15 & 1.95 & 0.58 & 0.36 \\
\hline V3 & 97.1 & -1.51 & 4.49 & 4.73 & -71.43 & 0.14 & 1.88 & 0.55 & 0.29 \\
\hline V4 & 96.5 & -1.63 & 5.56 & 5.79 & -73.62 & 0.17 & 1.94 & 0.99 & 0.17 \\
\hline V5 & 97.1 & -1.34 & 5.01 & 5.19 & -75.02 & 0.15 & 2.02 & 0.77 & 0.46 \\
\hline V6 & 96.6 & -1.80 & 4.59 & 4.92 & -68.62 & 0.16 & 1.76 & 0.00 & 0.00 \\
\hline
\end{tabular}

A perceptible colour difference between samples treated with enzyme preparations and control samples can be observed, suggesting that these products had a greater effect on colour. Delta values represent colour difference as compared to the control.

\subsection{Volatile compounds}

The aromatic profile of wines is influenced by numerous volatile compounds originating from the grapes or must, from yeast (fermentative aromas), or post-fermentative aromas (from aging processes) [11]. The strength and proportion of volatile substances set the category of wine odour. In Table 3, the most representative volatile compounds of analysed samples and their evolution during fermentation are represented.

Enzymatic treatments determine an increase in the total amount of volatile compounds compared to control samples. The results denote that the content of volatile compounds significantly fluctuate during fermentation and storage. Table 4 shows the concentration of volatile compounds identified in analysed wines and their odour descriptors.

Analysing V1 sample during fermentation, a significant increase of some compounds can be observed, such as: isoamylacetate, isoamylalcohol, ethyl caproate, 1-hexanol, ethyl octanoate, phenylethylacetate, hexanoic acid, benzeneethanol, octanoic acid. Also, other compounds registered a decrease, such as: 1-propanol, butanoic acid ethyl ester, ethyl decanoate, ethyl laurate, ethyl linoleolate, ethyl oleate. Variant V2 registered a relevant increase of isoamylalcohol, 3-ethoxy1-propanol, isovaleric acid, ethyl 4-hydroxybutanoate, isopropyl acetate, hexanoic acid, benzeneethanol, 3,7dimethyl-1,5-octadien-3,7-diol, and an important decrease of: ethyl caproate, ethyl decanoate, ethyl laurate, decanoic acid, ethyl linoleate, phenol, lauric acid, ethyl linoleolate, ethyl oleate. A significant increase of isoamylacetate, 1-butanol, isoamylalcohol, ethyl lactate, 1-hexanol, isobutyric acid, ethyl decanoate, isovaleric acid, hexanoic acid, octanoic acid, 4-vinyl-2-methoxyphenol, was noted in V3, with a decrease of ethyl octanoate and eicosane. V4 sample can be characterized by an important increase of some compounds, such as: 1-propanol, isoamylacetate, isoamylalcohol, hexanoic acid, benzeneethanol, 4-vinyl-2-methoxy-phenol, and a significant decrease of ethyl caproate, 1-hexanol, ethyl octanoate, ethyl decanoate, ethyl laurate, ethyl linoleolate. Isoamylalcohol, ethyl 4-hydroxybutanoate, hexanoic acid, benzeneethanol, octanoic acid and 4-vinyl2-methoxy-phenol showed an important increasing during alcoholic fermentation. Also, 1-hexanol, ethyl octanoate, 3-ethoxy-1-propanol, ethyl octanoate, eicosane, ethyl decanoate and isopropyl acetate were diminishined during fermentation.

Control sample showed a considerable decrease of isoamylalcohol, ethyl decanoate, decanoic acid, ethyl linoleate, ethyl oleate, and an important increase of isoamylacetate, 3-ethoxy-1-propanol, hexanoic acid, benzeneethanol, octanoic acid.

The most abundant compound in all samples is isoamylalcohol, followed by benzenethanol. By comparing the results of the V1, V2, V3, V4, V5 and V6 samples, of the 3 sampling days, the biggest differences are observed in case of fatty acids.

The concentration of the following esters derivatives decreases from the first day to the last day of sampling: ethyl caproate, ethyl octanoate, ethyl decanoate, ethyl laurate, ethyl palmitate, ethyl oleate, ethyl linoleate, ethyl linoleolate. Also, a decrease of fatty acids, such as decanoic acid and lauric acid, was registered during alcoholic fermentation. 
Table 3. Evaluation of volatile compounds during alcoholic fermentation (percent of total area).

\begin{tabular}{|c|c|c|c|c|c|c|c|c|c|c|c|c|c|c|c|c|c|c|c|}
\hline \multirow{2}{*}{ Volatile compounds } & \multirow{2}{*}{ R.T. } & \multicolumn{3}{|c|}{$\mathrm{V} 1$} & \multicolumn{3}{|c|}{$\mathrm{V} 2$} & \multicolumn{3}{|c|}{ V3 } & \multicolumn{3}{|c|}{ V4 } & \multicolumn{3}{|c|}{ V5 } & \multicolumn{3}{|c|}{ V6 } \\
\hline & & D 1 & D 3 & D 7 & D 1 & D 3 & D 7 & D 1 & D 3 & D 7 & D 1 & D 3 & D 7 & D 1 & D 3 & D 7 & D 1 & D 3 & D 7 \\
\hline 1-propanol & 11.46 & 1.26 & 1.86 & 0.98 & 1.34 & 1.54 & 1.68 & 1.23 & 1.91 & 1.73 & 1.61 & 2.36 & 2.47 & 2.71 & 2.12 & 2.03 & 2.34 & 2.28 & 2.41 \\
\hline $\begin{array}{l}\text { butanoic acid ethyl } \\
\text { ester }\end{array}$ & 11.56 & 0.21 & 0.40 & 0.11 & 0.30 & 0.48 & 0.43 & 0.28 & 0.43 & 0.35 & 0.32 & 0.40 & 0.43 & 0.47 & 0.31 & 0.44 & 0.29 & 0.40 & 0.36 \\
\hline isobutyl alcohol & 13.44 & 2.20 & 3.92 & 2.33 & 2.91 & 3.88 & 4.71 & 2.37 & 4.24 & 3.80 & 3.02 & 4.12 & 4.57 & 3.84 & 3.59 & 4.80 & 3.31 & 4.10 & 4.26 \\
\hline Isoamyl acetate & 14.91 & 1.14 & 1.91 & 4.02 & 1.37 & 2.14 & 2.00 & 1.04 & 1.89 & 2.51 & 1.62 & 2.38 & 2.16 & 1.38 & 1.87 & 1.79 & 1.44 & 2.02 & 2.44 \\
\hline 1-butanol & 15.64 & 0.88 & 0.12 & 0.11 & 0.13 & 0.15 & 0.16 & 0.08 & 0.15 & 0.17 & 0.12 & 0.16 & 0.15 & 0.14 & 0.16 & 0.17 & 0.15 & 0.10 & 0.13 \\
\hline dodecane & 18.16 & 0.13 & 0.21 & 0.14 & 0.07 & 0.18 & 0.13 & 0.09 & 0.20 & 0.06 & 0.16 & 0.19 & 0.22 & 0.20 & 0.23 & 0.23 & 0.37 & 0.21 & 0.13 \\
\hline isoamylalcohol & 18.54 & 27.17 & 41.26 & 31.28 & 30.88 & 44.06 & 46.51 & 26.83 & 42.58 & 47.23 & 39.57 & 44.63 & 49.40 & 48.88 & 48.49 & 53.02 & 43.50 & 48.51 & 33.49 \\
\hline ethyl caproate & 19.72 & 1.05 & 0.92 & 2.26 & 1.24 & 1.03 & 0.81 & 1.20 & 0.93 & 1.10 & 1.55 & 1.25 & 0.99 & 1.60 & 1.15 & 1.08 & 1.28 & 1.10 & 1.25 \\
\hline dotriacontane & 20.21 & 0.26 & 0.48 & 0.30 & 0.16 & 0.33 & 0.28 & 0.20 & 0.38 & 0.12 & 0.53 & 1.27 & 0.38 & 0.36 & 0.38 & 0.47 & 0.50 & 0.33 & 0.38 \\
\hline phytane & 22.55 & 0.19 & 0.31 & 0.22 & 0.15 & 0.27 & 0.28 & 0.17 & 0.31 & 0.12 & 0.25 & 0.46 & 0.31 & 0.21 & 0.32 & 0.39 & 0.62 & 0.23 & 0.34 \\
\hline ethyl lactate & 24.70 & 0.23 & 0.39 & 0.20 & 0.27 & 0.40 & 0.52 & 0.25 & 0.41 & 0.49 & 0.30 & 0.39 & 0.66 & 0.49 & 0.45 & 0.44 & 0.44 & 0.38 & 0.62 \\
\hline 1-hexanol & 24.98 & 0.49 & 0.71 & 0.94 & 0.59 & 0.82 & 0.82 & 0.54 & 0.76 & 1.05 & 1.15 & 0.89 & 1.08 & 1.10 & 0.85 & 0.77 & 1.19 & 1.03 & 1.15 \\
\hline $\begin{array}{l}\text { 3-ethoxy-1- } \\
\text { propanol }\end{array}$ & 26.03 & 0.74 & 1.16 & 0.07 & 0.79 & 1.09 & 1.20 & 0.75 & 1.17 & 0.89 & 1.00 & 1.16 & 1.27 & 1.48 & 0.88 & 1.23 & 0.85 & 1.30 & 1.47 \\
\hline ethyl octanoate & 28.59 & 4.37 & 3.42 & 8.50 & 3.82 & 3.14 & 2.33 & 4.62 & 2.98 & 2.71 & 3.40 & 3.05 & 2.49 & 3.24 & 2.36 & 1.78 & 3.23 & 2.08 & 2.99 \\
\hline eicosane & 28.98 & 0.41 & 0.60 & 0.37 & 0.39 & 0.54 & 0.65 & 0.42 & 0.63 & 0.29 & 0.66 & 1.85 & 0.64 & 0.62 & 0.50 & 0.47 & 0.89 & 0.49 & 0.73 \\
\hline acetic acid & 30.44 & 0.06 & 0.38 & 1.21 & 0.24 & 0.06 & 0.57 & 0.21 & 0.41 & 0.43 & 0.26 & 0.35 & 0.48 & 0.45 & 0.39 & 0.64 & - & 0.29 & 0.56 \\
\hline isobutyric acid & 35.12 & 0.09 & 0.14 & - & 0.10 & 0.13 & 0.17 & 0.08 & 0.13 & 0.11 & 0.10 & 0.13 & 0.13 & 0.16 & 0.09 & 0.10 & 0.06 & 0.09 & 0.16 \\
\hline ethyl decanoate & 36.92 & 7.41 & 2.73 & 5.51 & 5.81 & 1.97 & 1.43 & 7.78 & 2.28 & 0.97 & 3.60 & 2.78 & 1.05 & 2.53 & 1.18 & 1.29 & 3.19 & 2.06 & 1.77 \\
\hline butanoic acid & 37.50 & 0.16 & 0.27 & 0.20 & 0.20 & 0.29 & 0.32 & 0.15 & 0.26 & 0.18 & 0.23 & 0.44 & 0.23 & 0.27 & 0.25 & 0.28 & 0.15 & 0.32 & 0.38 \\
\hline isovaleric acid & 39.02 & 0.12 & 0.27 & 0.13 & 0.12 & 0.29 & 0.36 & 0.11 & 0.21 & 0.25 & 0.22 & 0.27 & 0.27 & 0.27 & 0.20 & 0.21 & 0.10 & 0.21 & 0.41 \\
\hline $\begin{array}{l}\text { ethyl } \\
\text { hydroxybutanoate }\end{array}$ & 43.30 & 0.64 & 1.17 & 0.26 & 0.73 & 1.23 & 1.35 & 0.58 & 1.19 & 1.08 & 0.86 & 1.29 & 1.13 & 0.75 & 0.94 & 1.02 & 0.77 & 0.99 & 1.63 \\
\hline ethyl laurate & 44.52 & 5.24 & 0.67 & 1.89 & 4.37 & 0.46 & 0.20 & 4.80 & 0.54 & 0.07 & 2.17 & 0.42 & - & 0.79 & 0.26 & 0.16 & - & - & - \\
\hline 1,3-dithiolane & 40.13 & 0.11 & 0.17 & 0.06 & 0.09 & 0.16 & 0.18 & 0.07 & 0.14 & 0.12 & 0.10 & - & - & 0.14 & 0.12 & 0.13 & - & 0.11 & 0.21 \\
\hline isopropyl acetate & 40.92 & 1.41 & 2.22 & 1.48 & 1.60 & 2.27 & 2.39 & 1.48 & 2.30 & 1.77 & 2.04 & 1.72 & 2.62 & 2.21 & 1.81 & 1.87 & 1.61 & 1.71 & 2.93 \\
\hline phenylethylacetate & 43.69 & 0.12 & 0.25 & 0.41 & 0.11 & 0.18 & 0.18 & 0.13 & 0.25 & 0.13 & 0.17 & 0.17 & 0.18 & 0.13 & 0.19 & 0.25 & 0.30 & 0.15 & 0.27 \\
\hline hexanoic acid & 45.40 & 1.05 & 1.81 & 2.73 & 1.21 & 2.13 & 2.63 & 1.17 & 2.18 & 2.95 & 1.77 & 1.95 & 2.28 & 1.54 & 1.74 & 2.02 & 1.68 & 2.71 & 3.47 \\
\hline butyl acetate & 45.81 & 0.35 & 0.63 & 0.04 & 0.34 & 0.52 & 0.54 & 0.35 & 0.55 & 0.41 & 0.50 & 0.43 & 0.47 & 0.57 & 0.43 & 0.53 & 0.70 & 0.66 & 0.82 \\
\hline benzeneethanol & 47.19 & 7.16 & 11.11 & 7.91 & 8.39 & 12.18 & 13.61 & 7.59 & 12.18 & 15.79 & 10.89 & 11.78 & 12.44 & 9.99 & 13.62 & 11.38 & 9.84 & 10.31 & 16.81 \\
\hline octanoic acid & 52.51 & 1.76 & 3.42 & 5.45 & 2.41 & 4.01 & 4.84 & 2.18 & 4.05 & 6.06 & 3.35 & 3.78 & 4.31 & 2.63 & 5.13 & 3.96 & 4.79 & 3.86 & 6.48 \\
\hline $\begin{array}{l}\text { 3,7-dimethyl-1,5- } \\
\text { octadien-3,7-diol }\end{array}$ & 48.12 & 0.05 & - & 0.17 & 0.06 & 0.10 & 0.12 & 0.05 & 0.10 & 0.07 & 0.09 & 0.11 & 0.13 & 0.08 & 0.13 & 0.08 & - & - & - \\
\hline $\begin{array}{l}\text { 4-vinyl-2- } \\
\text { methoxy-phenol }\end{array}$ & 56.46 & 0.20 & 0.63 & - & 0.24 & 0.68 & 1.03 & 0.21 & 0.62 & 1.24 & 0.39 & 0.87 & 1.06 & 0.32 & 0.91 & 0.89 & - & 0.65 & 1.43 \\
\hline decanoic acid & 59.01 & 1.50 & 1.21 & 4.51 & 1.77 & 1.32 & 1.12 & 1.99 & 1.32 & 1.67 & 1.93 & 1.18 & 1.18 & 1.21 & 1.61 & 1.39 & 2.94 & 1.24 & 1.80 \\
\hline 4-vinylphenol & 62.35 & - & 0.49 & 0.21 & - & 0.58 & 0.80 & - & 0.54 & 0.97 & - & 0.47 & 0.36 & - & 0.41 & 0.63 & - & 0.34 & - \\
\hline ethyl linoleate & 65.55 & 10.44 & 1.54 & 0.30 & 8.60 & 0.77 & - & 9.95 & 1.07 & - & 4.03 & 0.76 & - & - & - & - & 2.58 & 0.59 & - \\
\hline $\begin{array}{l}\text { phenol, 2,4- } \\
\text { bis(1,1-dimethyl) }\end{array}$ & 59.76 & 0.39 & 0.59 & 0.47 & 0.32 & 0.48 & 0.57 & 0.35 & 0.50 & 0.20 & 0.95 & - & 0.48 & 0.40 & 0.57 & 0.64 & 0.77 & 0.41 & 0.64 \\
\hline ethyl oleate & 64.21 & 2.19 & 0.35 & 0.12 & 1.94 & 0.16 & - & 2.06 & 0.22 & - & 0.78 & 0.16 & - & 0.54 & 0.23 & - & 0.44 & 0.20 & - \\
\hline lauric acid & 64.95 & 1.20 & 0.27 & 2.77 & 0.95 & 0.32 & - & 1.38 & 0.15 & - & 0.70 & 0.18 & - & 0.33 & 0.26 & - & 1.10 & 0.13 & - \\
\hline ethyl linoleolate & 67.37 & 3.65 & 0.62 & 0.23 & 3.50 & 0.50 & - & 1.47 & 0.36 & - & 1.47 & 0.36 & - & 0.73 & 0.53 & - & 1.07 & 0.32 & - \\
\hline stearic acid & 72.11 & - & 4.18 & 0.83 & - & 3.52 & 1.18 & - & 3.04 & 1.32 & - & 0.73 & 1.07 & - & - & 1.03 & 1.05 & 0.81 & 2.75 \\
\hline
\end{tabular}


Table 4. Percent concentration of volatile compounds identified in analysed wines and their odour descriptors.

\begin{tabular}{|c|c|c|c|c|c|c|c|c|c|c|}
\hline \multirow{2}{*}{ No. } & \multirow{2}{*}{ Compound name } & \multirow{2}{*}{ RT } & \multicolumn{6}{|c|}{ FINAL WINES PERCENT OF TOTAL AREA } & \multirow{2}{*}{ Odour descriptors } & \multirow{2}{*}{ Ref. } \\
\hline & & & V1 & V2 & V3 & V4 & V5 & V6 & & \\
\hline \multicolumn{11}{|c|}{ ALCOHOLS } \\
\hline 1 & 1-propanol & 11.46 & 0.50 & 1.97 & 1.63 & 2.11 & 2.23 & 1.95 & Fruity & [5] \\
\hline 2 & 1-butanol & 15.64 & 0.17 & 0.12 & 0.15 & 0.14 & 0.12 & 0.16 & Fusel, spirits & {$[5]$} \\
\hline 3 & 1-hexanol & 24.97 & 1.22 & 0.84 & 0.81 & 0.95 & 0.78 & 0.64 & $\begin{array}{l}\text { Vegetal, green, fruity, } \\
\text { apple-skin, oily }\end{array}$ & {$[12][13]$} \\
\hline 4 & $\begin{array}{l}\text { isoamylalcohol } \\
\text { (isopentyl alcohol) }\end{array}$ & 18.53 & 37.18 & 41.82 & 42.39 & 45.67 & 45.50 & 49.88 & Harsh, nail polish, banana & {$[5]$} \\
\hline 5 & $\begin{array}{l}\text { benzeneethanol } \\
\text { (Phenylethyl alcohol) }\end{array}$ & 47.19 & 13.50 & 12.85 & 12.78 & 12.64 & 15.25 & 10.79 & Floral, rose & {$[5]$} \\
\hline 6 & 3-penten-2-ol & 16.83 & 0.09 & 0.12 & 0.09 & 0.11 & 0.11 & 0.14 & Kiwi, fruity, acrid, pungent & {$[14]$} \\
\hline 7 & 3-ethoxy-1-propanol & 26.02 & 0.68 & 1.09 & 1.13 & 1.21 & 0.89 & 1.21 & Fruity & [15] \\
\hline \multicolumn{11}{|c|}{ ESTERS } \\
\hline 8 & ethyl octanoate & 28.59 & 2.33 & 1.67 & 1.80 & 1.90 & 1.66 & 2.22 & $\begin{array}{l}\text { Fruity, banana, apple, } \\
\text { pineapple, pear, floral, } \\
\text { sweet, soap }\end{array}$ & [12] [5] [15] \\
\hline 9 & ethyl caproate & 19.73 & 1.15 & 0.71 & 0.70 & 0.78 & 0.64 & 0.92 & Fruity, pineapple-banana & [12] \\
\hline 10 & ethyl lactate & 24.70 & 0.95 & 1.86 & 1.92 & 2.07 & 2.15 & 1.57 & Ethereal, buttery odour & [16] \\
\hline 11 & ethyl 3-hydroxy butyrate & 32.23 & 0.15 & 0.34 & 0.34 & 0.32 & 0.39 & 0.39 & Fresh fruits & [15] \\
\hline 12 & butanoic acid ethyl ester & 11.57 & 0.19 & 0.34 & 0.42 & 0.44 & 0.35 & 0.42 & - & - \\
\hline 13 & $\begin{array}{l}\text { isoamylacetate } \\
\text { (3-Methylbutyl acetate) }\end{array}$ & 14.91 & 3.60 & 1.25 & 1.11 & 1.40 & 1.07 & 1.33 & Fruity, banana, solvent, pear & {$[12][5]$} \\
\hline 14 & ethyl decanoate & 36.92 & 1.85 & 1.15 & 1.44 & 1.26 & 1.07 & 1.38 & Floral, soap, fatty & [5] \\
\hline 15 & $\begin{array}{l}\text { diethyl } \\
\text { hydroxybutanedioate } \\
\text { (Diethyl tartrate) }\end{array}$ & 51.48 & 0.24 & 0.46 & 0.44 & 0.53 & 0.54 & 0.27 & - & - \\
\hline 16 & $\begin{array}{l}\text { butanedioic acid diethyl } \\
\text { ester (Diethyl succinate) }\end{array}$ & 38.48 & 0.31 & 0.43 & 0.42 & 0.44 & 0.46 & 0.51 & Faint, pleasant odour & {$[12]$} \\
\hline 17 & $\begin{array}{l}\text { butandienoic acid, diethyl } \\
\text { ester }\end{array}$ & 63.18 & 2.41 & 2.41 & 2.47 & 2.88 & 3.04 & 3.31 & $\begin{array}{l}\text { Fruity, waxy, floral, slightly } \\
\text { musty }\end{array}$ & {$[16]$} \\
\hline 28 & ethyl 4-hydroxybutanoate & 43.30 & 2.18 & 0.92 & 0.90 & 0.80 & 0.89 & 0.78 & - & \\
\hline 19 & phenylethylacetate & 43.76 & 0.33 & - & - & - & - & - & $\begin{array}{l}\text { Honey, floral, rose, green, } \\
\text { hay, cocoa }\end{array}$ & {$[13]$} \\
\hline 20 & 1-hexyl acetate & 21.46 & 0.15 & - & - & - & - & - & $\begin{array}{l}\text { Fruity, fresh, green, sweet, } \\
\text { apple, pear, banana peel }\end{array}$ & {$[13]$} \\
\hline 21 & isopropyl acetate & 40.91 & 1.08 & 1.87 & 1.90 & 1.53 & 1.60 & 2.16 & - & - \\
\hline 22 & butyl acetate & 45.80 & 0.34 & 0.41 & 0.46 & 0.42 & 0.48 & 0.38 & Fruity, pineapple & [16] \\
\hline \multicolumn{11}{|c|}{ ACIDES } \\
\hline 23 & acetic acid & 30.49 & 2.59 & 0.57 & 0.56 & 0.60 & 0.59 & 0.69 & Green, sour, rancid & [9] \\
\hline 24 & butanoic acid & 37.54 & 0.32 & 0.29 & 0.27 & 0.29 & - & 0.33 & Animal & [12] \\
\hline 25 & $\begin{array}{l}\text { isovaleric acid } \\
\text { (3-methyl butanoic acid) }\end{array}$ & 39.07 & 0.18 & 0.31 & 0.31 & 0.31 & 0.28 & 0.36 & Rancid, cheese, rotten fruits & [17] \\
\hline 26 & isobutyric acid & 35.15 & 0.15 & 0.13 & 0.14 & 0.15 & 0.11 & 0.18 & $\begin{array}{l}\text { Pungent, ethereal, fruity, } \\
\text { rum-like nuance }\end{array}$ & {$[16]$} \\
\hline 27 & hexanoic acid & 45.40 & 4.28 & 2.42 & 2.45 & 2.09 & 1.93 & 2.31 & Rancid, fatty & [17] \\
\hline 38 & octanoic acid & 52.52 & 8.18 & 4.44 & 4.29 & 5.19 & 4.74 & 3.63 & $\begin{array}{l}\text { Fatty acid, dry, dairy, } \\
\text { cheese, sweet }\end{array}$ & {$[17]$} \\
\hline 29 & stearic acid & 72.16 & - & 2.03 & 1.97 & 2.17 & 2.21 & 1.66 & - & - \\
\hline 30 & decanoic acid & 59.04 & 2.19 & 0.89 & 0.93 & 1.05 & 1.06 & 0.73 & Rancid, fat, dry, woody & [9] \\
\hline
\end{tabular}

*Ref - References. 


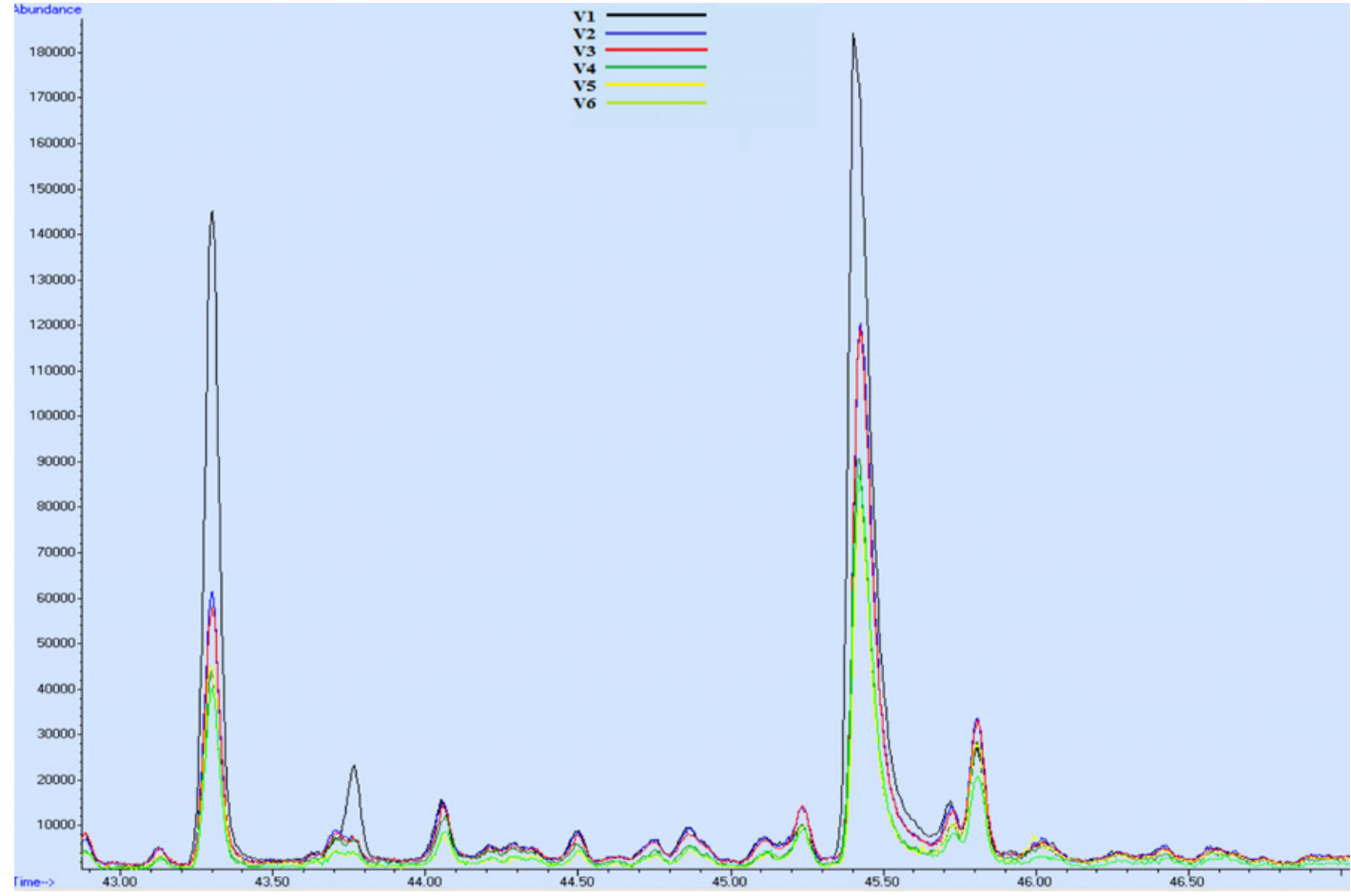

Figure 1. GC chromatogram for distribution of volatile components in analyzed wines.

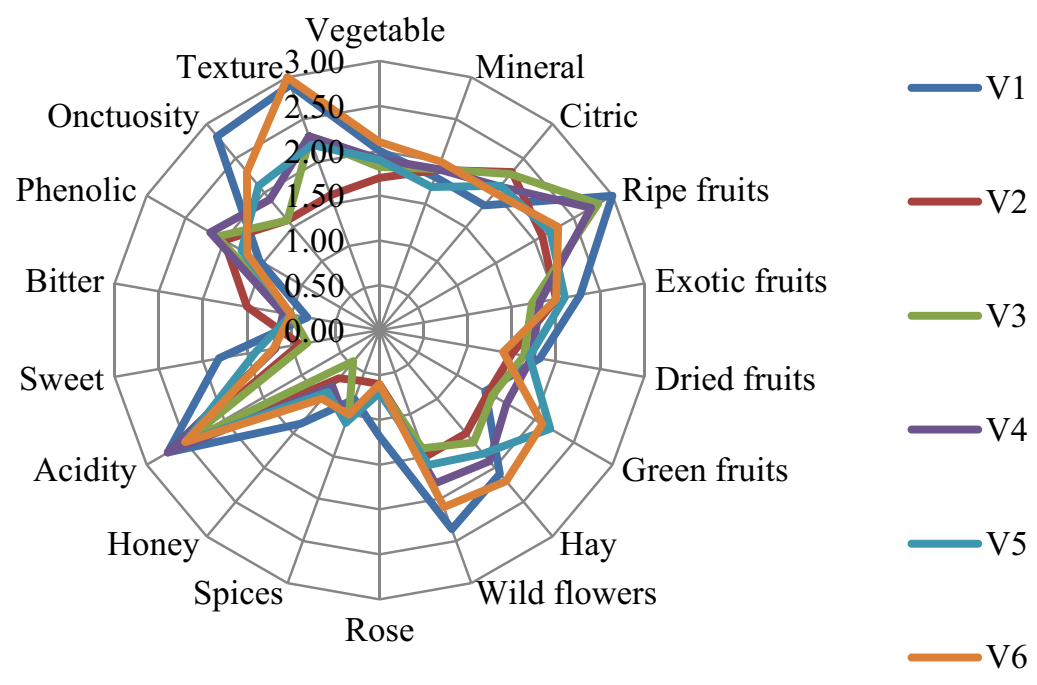

Figure 2. Organoleptic parameters of obtained wines.

These differences can be generated by enzymes reactions and yeast. Saccharomyces cerevisiae yeast can have a major contribution to wine aroma profile.

The aroma profile of analysed samples is expressed especially by higher alcohols, esters and acids. The highest concentration in alcohols was detected in V5 variant, followed by V6, V3, V2, and V1. On the other hand, the richest variant in esters was V1, followed by V6, $\mathrm{V} 4, \mathrm{~V} 3, \mathrm{~V} 2$ and V5. V1 variant registered the highest amount of acids, followed by V4, V3, V5, V2 and V6. Isoamylalcohol and benzenethanol were the highest among the total of identified alcohols, imprinting complexity to the aroma. Esters constitute one of the most important classes of aroma compounds, with an important effect on the fruity notes in wines (tropical fruits, banana, pineapple, pears). Butandienoic acid diethyl ester showed a high concentration in all variants. Organic acids enhance bitterness, freshness and astringency. Butanoic, hexanoic and decanoic acids presented the highest proportion. Phenylethylacetate and 1-hexyl acetate are specific for V1 sample (treated with Endozym Thiol ${ }^{\circledR}$ ).

Significant differences can be observed referring to the quality of final wines, reported on the type of used enzyme. Enzymes released additional volatile compounds from the grapes, reducing the amount of alcohol, allowing a better perception and release of aroma compounds. The different distribution of volatile components in analysed wines is presented in Fig. 1. 


\subsection{Sensory characteristics}

The final sensorial quality of wine is the result of a multitude of interaction between all the volatile components. The character of wine is influenced by the sweetness, acidity and astringency/bitterness, generated by non-volatile substances soluble in water or alcohol mixture. Together with volatile compounds, they are liable for the general aroma profile. Perceptible sweetness is significantly determined by numerous constituents, such as ethanol, acids or tannins. Detectable sweetness presents a diminishing effect on sourness and bitterness [18]. Sweet note was better remarked at V1 sample.

The principal organoleptic parameters of analysed wines are presented in Fig. 1. Following the sensory analysis, significant differences can be observed due to the type of enzyme preparation used as pre-treatment.

Enzymatic treatments generated an increase in flavor descriptors content compared to control sample. All wines were described as equilibrated, with a great acidity, that imprinted freshness and a good texture.

Control sample was characterized by hay notes, honey and vegetable flavour. V1 sample was remarked for its fruity taste (ripe fruits, exotic fruits, dry fruits), wild flowers and roses nose. Citric flavour was dominant in V2 sample and green fruits aroma is prevalent in V5.

Regarding the correlation between the chemical compounds and the results of sensorial analysis, the fruity notes (ripe fruits) of samples can be explained by the presence of 1-propanol, 1-hexyl acetate, 1-hexanol, ethyl octanoate, ethyl caproate. The presence of 1-hexanol explains the vegetal notes of samples. Ethyl caproate and isoamylacetate are the main responsible for the tropical aromas.

\section{Conclusions}

In this study, the pre-fermentative treatments did not appear to have a significant influence on the basic physicalchemical parameters. A perceptible colour difference between samples treated with enzyme preparations and control samples was observed, suggesting that enzymes preparation had a major effect on colour. Following the evolution of volatile compounds during fermentation, enzymes released additional volatile compounds from the grapes, reducing the amount of alcohol, thus allowing for a better perception and release of aroma compounds. Enzymatic treatments determine an increase in the total amount of volatile compounds compared to control sample. The most abundant compound in all samples is isoamylalcohol, followed by benzenethanol. Significant differences can be observed referring to the quality of final wines and on the type of used enzyme. The aroma profile of analysed samples is expressed especially by higher alcohols, esters, and acids. Following the sensory analysis, significant differences can be observed due to the type of enzyme preparation used as pre-treatment.

Enzymes are effective, specific and convenient to use and the need for a more in depth search is clear.

We thank "Iuliu Haţieganu" University of Medicine and Pharmacy, Cluj-Napoca, Romania and Research Center for Oenology, Romanian Academy - Iaşi Branch for the scientific support. The research was funded by the FDI project, registration final code CNFIS-FDI-2019-0267.

\section{References}

[1] K.D. Mojsov, D. Andronikov, A. Janevski, S. Jordeva, S. Zezova, Adv. technol. 4, 94 (2015)

[2] E. Gómez-Plaza, I. Romero-Cascales, A.B. BautistaOrtin, Enzymes in Fruit and Vegetable ProcessingUse of Enzymes for Wine Production (CRC Press, 2010)

[3] E. Zarzoso, Int. Microbiol. (1999)

[4] P. Romano, A. Capece, L. Jespersen, Springer Sci. 2 (2006)

[5] J.H. Swiegers, E.J. Bartowsky, P.A. Henshke, I.S. Pretorius, Aust J Grape Wine R 11, 139 (2005)

[6] H. Claus, K. Mojsov, J. of Ferment. Technol. 4, 52 (2018)

[7] M. Ugliano, Springer Sci. Wine Chem. Biochem. (2009)

[8] Organisation International de la Vigne et du Vin, Compendium of international methods of analysis (2013)

[9] G.L. Main G.L., J.R. Morris, AJEV 58, 3 (2007)

[10] J. Samoticha, A. Wojdyło, J. Chmielewska, I. Politowicz, A. Szumny, JFST 79, 445, (2017)

[11] D.V Cotea, Ceres Print, Oenology Treatise (1985)

[12] K. Mojsov, IJTMkt 9, 112 (2013)

[13] R.J. Bakker, J. Clarke, Wine Flavour Chemistry (Blacwell Publishing, 2004)

[14] F. Vararu, J. Moreno-Garcia, V.V. Cotea, J. Moreno, Vitis J. 54 (2015)

[15] Y.H. Hui, Feng Chen, M. L. Leo. Handbook of Fruit and Vegetable Flavors (Canada: Jon Wiley and Sons Publication, 2010)

[16] R. Velàzquez, E. Zamora, L. Maria Álvarez, L.M. Hernándes, M. Ramirez, Front. Microbiol. 6, 1222 (2016)

[17] G.A. Burdock, Fenaroli's Handbook of Flavor Ingredients (CRC Press, 2010)

[18] R.G. Berger, Springer Sci. (2007)

[19] R.S Jackson. Wine Science - Principles and Applications (2008) 